\title{
An array of quantum dots as a spin filter device by using Dicke and Fano effects
}

\author{
Pedro A. Orellana ${ }^{1}$, Judith Ojeda ${ }^{2}$, Monica Pacheco $^{2}$ \\ ${ }^{1}$ Departamento de Física, Universidad Católica del Norte, \\ Avenida Angamos 0610, Antofagasta, Chile \\ ${ }^{2}$ Departamento de Física, Universidad Técnica Federico Santa María, \\ Casilla 110-V, Valparaiso Chile \\ orellana@ucn.cl
}

Recently, there has been much interest in understanding the manner in which the unique properties of nanostructures may be exploited in spintronics devices, which utilize the spin degree of freedom of the electron as the basis of their operation [1-2]. A natural feature of these devices is the direct connection between their conductance and their quantum-mechanical transmission properties, which may allow their use as an all-electrical means for generating and detecting spin polarized distributions of carriers.

Quantum dots are man-made nanostructures in which electrons are confined in all space dimensions [3]. Energy and charge quantization results from this confinement. As both features are present in real atomic systems, useful analogies between real and artificial atomic systems have been exploited recently. Enforcing this analogy, Fano [4-5] and Dicke effects [6] were also found to be present in quantum dot configurations. On the other hand, Song et al. [2] described how a spin filter may be achieved in open quantum dot systems, by exploiting the Fano resonances that occur in their transmission characteristic. In a quantum dot in which the spin degeneracy of carrier is lifted they showed that the Fano effect may be used as an effective means to generate spin polarization of transmitted carriers and that electrical detection of the resulting polarization should be possible.

In a previous work [7], we showed that in side-coupled double quantum-dot system the transmission shows a large peak-to-valley ratio. Moreover, the difference of energy between the resonances and antiresonances can be controlled adjusting the difference between the energy levels of the two quantum dots by gate voltages due to the Dicke effect. The Dicke effect in optics takes place in the spontaneous emission of a pair of atoms radiating a photon with a wavelength much larger than the separation between them [6]. The luminescence spectrum is characterized by a narrow and a broad peak, associated with long and short-lived states, respectively. The former state, coupled weakly to the electromagnetic field, is called subradiant, and the latter, strongly coupled, a superradiant state. In the present work, we show that the above properties can be used to design an efficient spin filter with a quantum dot array system. We show that by controlling the Fano and Dicke effects this system can be used as an efficient spin filter even for small values of the magnetic field.

In this work we propose a new design of a spin-dependent polarizer. The device consists of a quantum dot array coupled to leads, as shown schematically in Fig1. By lifting the spin degeneracy of the carriers in a quantum dot by means of a magnetic field, Fano and Dicke effects are used as effective means to generate spin polarization of the electrical current. A detailed analysis of the spin-dependent transmission and polarized current is carried out. For instance, in Fig. 2 we show a contour plot with the weighted spin polarization for a fixed magnetic field of as a function of the Fermi energy and $\Delta$ the asymmetric parameter. The region of maximum of polarization in centered on the Zeeman energy and it grows with $\Delta$. 


\section{References:}

[1]S. Datta and B. Das, Appl. Phys. Lett. 56, (1990) 665.

[2] J. F. Song, Y. Ochiai and J. P. Bird, Appl. Phys. Lett. 82 (2003) 4561.

[3] L.Jacak, P.Hawrylak and A.Wojs, Quantum Dots (Springer--Verlag, Berlin, 1998).

[4] U. Fano, Phys. Rev. 124, (1961) 1866.

[5] K. Kobayashi, H. Aikawa, A. Sano, S. Katsumoto and Y. Iye, Phys. Rev. B 70, (2004) 035319.

[6] Dicke R H, Phys. Rev. 89 (1953) 472.

[7] P. A. Orellana and F. Domínguez-Adame, phys.stat. sol. (a) 203, (2006) 1178.

\section{Figures:}

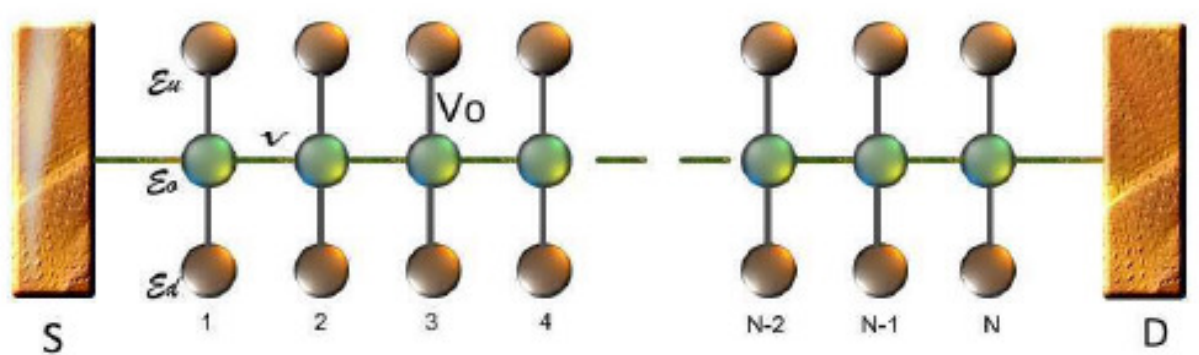

Fig 1: Schematic view of a quantum dot array coupling to leads.

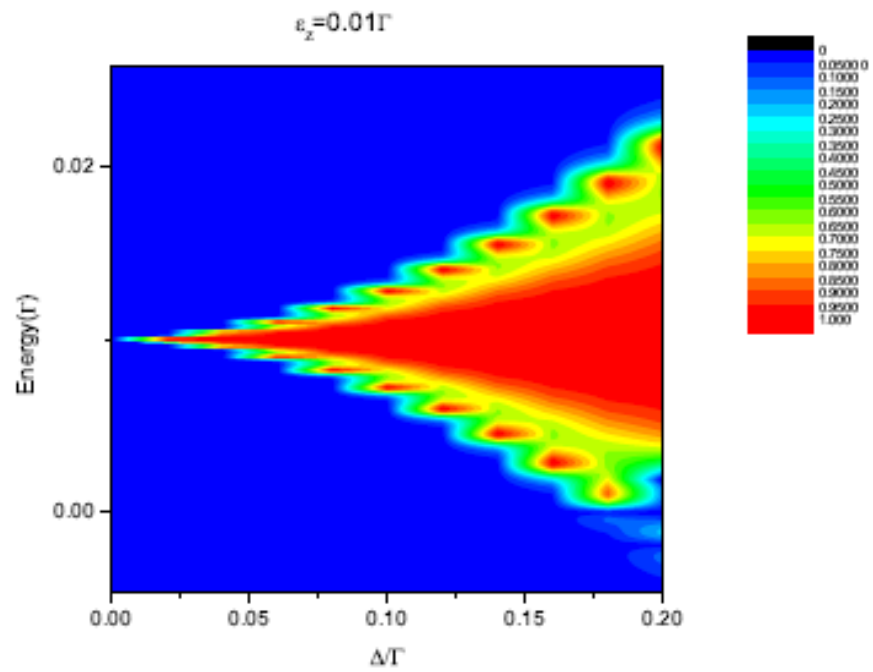

Fig 2: Contour plot of weighted spin polarization for the quantum dot array as a function of the Fermi energy and $\Delta$ for fixed magnetic field. 